\title{
Los Caminos del Agua y la Construcción de Terrazas Agrícolas EN EL PaÍs de la LLUVIa
}

\section{Water Paths and THE CONSTRUCTION OF Agricultural TERRACING IN A LAND OF RAIN}

\author{
ÁNGELES SANTOS VÁZQUEZ \\ angeles.santos@udc.es \\ HENRIQUE SEOANE \\ enrique.seoane@udc.es \\ CARLOS MARTÍNEZ GONZÁLEZ \\ c.martinez.gonzalez@udc.es \\ UNIVERSIDADE DA CORUÑ ${ }^{1}$
}

[RECIBIDO 24/12/2019; ACEPTADO 23/06/2020]

http://doi.org/10.25267/Riparia.2020.v6.04

\section{RESUMÉN}

El hábitat del territorio de la antigua Gallaecia en el noroeste peninsular se caracteriza por la diseminación de la población en varias decenas de miles de pequeñas aldeas dispersas geográficamente que modelaron a su alrededor un territorio propio, asociado a su propia dinámica evolutiva, pero con raíces en un remoto pasado derivado de procesos de sedentarización que decantaron en la cultura de los castros. El estudio

\footnotetext{
1 Ángeles Santos Vázquez: Profesora asociada del Departamento de Construcións e Estructuras Arquitectónicas, Civís e Aeronáuticas. Grupo de investigación Sistemas Constructivos y Rehabilitación (SICOR). Henrique Seoane: Profesor asociado del Departamento de Proxectos Arquitectónicos, Urbanismo e Composición. Grupo de investigación Galicia Ciudad y Territorio (GCéT). Carlos Martínez González: Profesor contratado interino del Departamento de Proxectos Arquitectónicos, Urbanismo e Composición. Grupo de investigación Paisaje, Arquitectura y Ciudad (pARQc). Universidade da Coruña. Escola Técnica Superior de Arquitectura, Campus da Zapateira, 15071 A Coruña.
}

A. Santos Vázquez, H. Seoane, C. Martínez González, "Los caminos del agua y la construcción de terrazas agrícolas en el país de la lluvia", RIPARIA 6 (2020), 151-179. 
pormenorizado de la aldea de A Burgueira y su territorio adscrito, emplazada en el tramo alto del valle del río Tamuxe, en la comarca del Rosal, sirve de paradigma para describir como las decisiones de asentamiento se han tomado comunitariamente desde un profundo conocimiento del medio, y en función de las posibilidades que ofrece el agua, necesaria para la vida, que manejada y administrada sabiamente induce y condiciona la sucesiva formalización de terrazas territoriales, cuya construcción ha favorecido un intenso y tradicional laboreo agrícola.

Palabras Claves: Territorio hídrico, Aldea, Terrazas Agrícolas, Regadío Tradicional, Procomún

ABSTRACT The habitat of the territory of ancient Gallaecia in the NorthWest of the Iberian peninsula is characterized by the distribution of its population throughout tens of thousands of small hamlets which have configured their environment in such a way as to form their own distinctive territory, associated with its evolutionary dynamic with roots in a remote past of patterns of human settlement that culminates in the culture of the castros. This study describes the hamlet of A Burgeira and its associated territory which is located in the upper levels of the River Tamuxe valley in the district of Rosal. It can serve as a paradigm of how decisions concerning the location for a settlement were taken communally employing a profound knowledge of the environment with regard to the availability of water resources. These resources, managed wisely, have conditioned the successive elaborations of the terracing which have enabled intense traditional cultivation.

KEY WORDS: Hydric Territory, Hamlet, Agricultural Terraces, Traditional Irrigation, The Common 


\section{Introducción}

Galicia, un territorio situado al noroeste de la Península Ibérica, forma parte de la Europa húmeda y como región oceánica se sitúa entre las más lluviosas de la Europa occidental. La precipitación anual ponderada de Galicia es de $1.180 \mathrm{~mm}^{2}$. Con más de 21.200 ríos sobre la corteza de este singular territorio, que a su vez generan una tupida red de $32.000 \mathrm{Km}$ de cursos de agua uniformemente repartidos por su geografía $-29.600 \mathrm{Km}^{2}$ de superficie -dan como resultado una densidad de 1,08 Km por $\mathrm{Km}^{2}$ de líneas, corrientes superficiales de agua que drenan un territorio en el que resulta muy difícil caminar en cualquier dirección más de un kilómetro sin encontrarse con una corriente continua de agua ${ }^{3}$.

Quizás esta sea una de las razones que explican que Galicia sea el territorio de las aldeas ${ }^{4}$. Más de 30.000 pequeñas aldeas dispersas y repartidas uniformemente caracterizan un territorio completa e intensamente antropizado, resultado de un proceso iniciado con la cultura de los castros en la Edad de Hierro y que hoy presenta una densidad de más de un núcleo de población por $\mathrm{Km}^{2}$. La densa red hidrológica que caracteriza estas tierras tiene que ver con la abundancia de las lluvias y el carácter impermeable de las rocas que forman el sustrato ${ }^{5}$, fundamentalmente, granitos y esquistos en la zona de estudio ${ }^{6}$. Rocas impermeables que solo adquieren una cierta tasa de permeabilidad dependiendo del desarrollo que alcancen los procesos de fracturación, meteorización y disolución.

\footnotetext{
2 A. Martinez Cortizas, A. Perez Alberti, Atlas Climático de Galicia, Santiago de Compostela 1999, 16 y 79.

${ }^{3}$ E. SEOANE, A ría como soporte da construcción da periferia urbana, A Coruña 2013, 12.

4 IBIDEM, 21.

5 F. Carreras y Candi, Geografía General del Reino de Galicia, 1980, 4.

6 Fuente: Mapa Litológico de Galicia, Instituto de Estudos do Territorio (IET) http://mapas.xunta.gal/portada
} 
Prácticamente el 90\% del territorio de Galicia presenta acuíferos en medios fracturados ${ }^{7}$.

Se presenta el estudio de una aldea situada en el sur de Galicia, concretamente en el Valle del Rosal, a los pies de A Serra da Groba, una sierra litoral con elevaciones entre 500 y 600 metros, y que limita con las tierras hermanas del norte de Portugal, río Miño por medio.

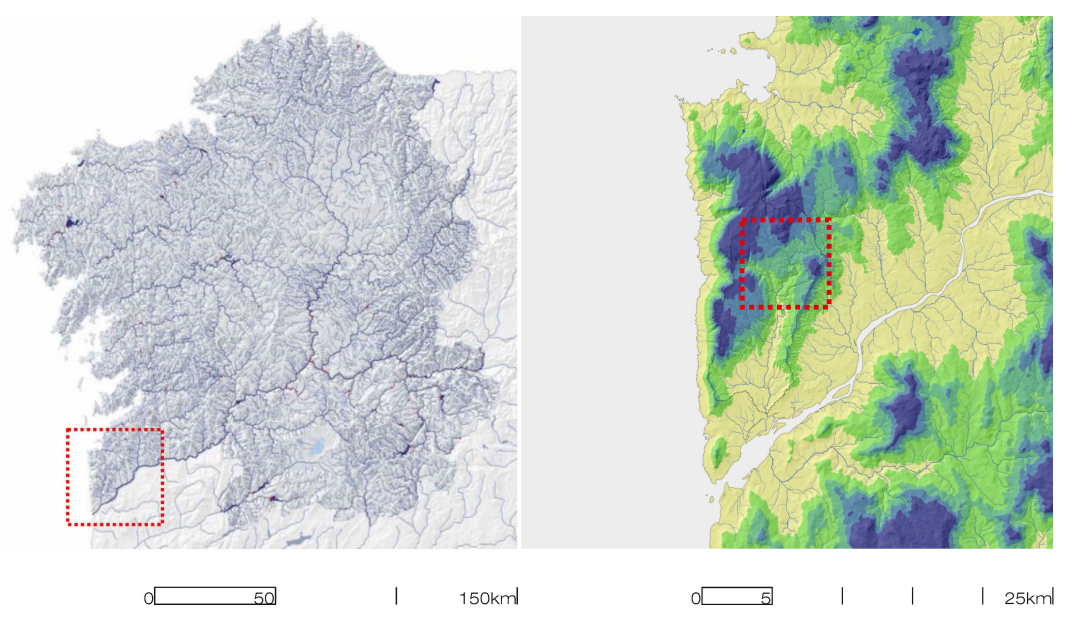

Fig. 1. Galicia: Elaboración propia ${ }^{8}$

Las sierras litorales, situadas en la línea de costa, son los primeros obstáculos que se encuentran los vientos del Oeste, de procedencia oceánica, y cargados con una elevada cuota de humedad que las convierten en áreas de máximos pluviométricos,

\footnotetext{
7 J. R. Raposo, J. Moreno, J. Dafonte, "Parameterization and quantification of recharge in crystalline fractures bedrocks in Galicia-Costa (NW Spain)". Hydrol, Earth, $16,2012,1667-1683$.

8 "Galicia, con una superficie en torno a $220 \mathrm{~km}$ por $220 \mathrm{~km}$ cuenta con 21.200 corrientes de agua distribuidas uniformemente. El recuadro rojo señala la zona de estudio". En la parte de la derecha se observa el Valle del Rosal, protegido por las elevaciones montañosas de A Serra da Groba. En este valle se señala el territorio de 3,5 x 3,5 km donde se sitúa la aldea de A Burgueira.
}

"Los caminos del agua y la construcción de terrazas agrícolas..." 
con medias de 1800-2000 mm . Estas montañas litorales, donde brotan manantiales y ríos, permiten disponer de agua de superficie en abundancia. En el norte de Portugal reciben el nombre de "castillos de agua"10.

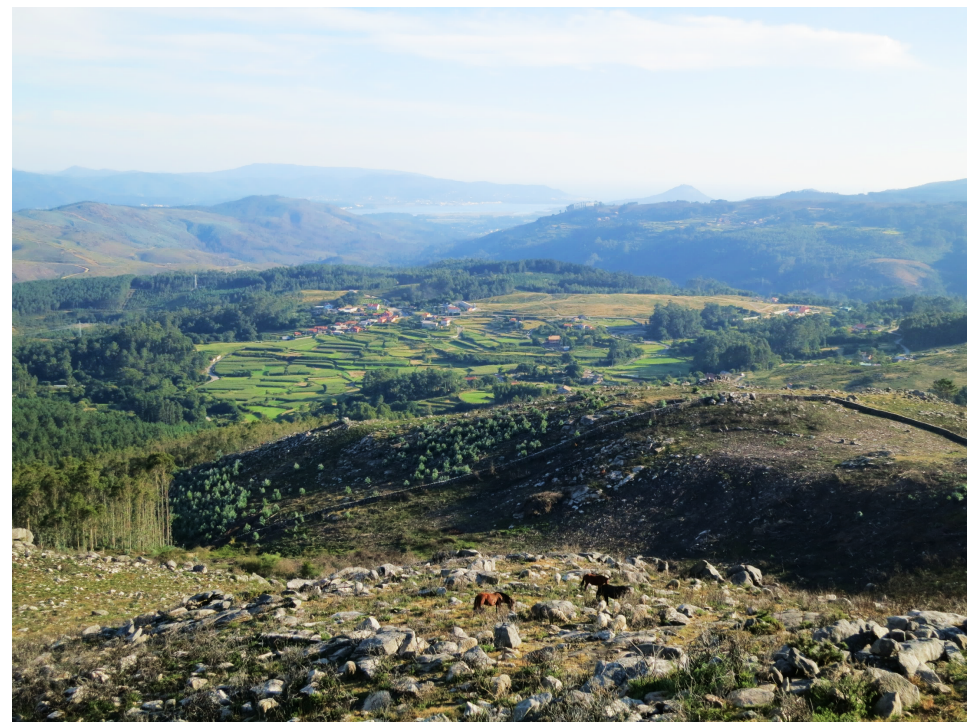

Fig. 2. Aldea de A Burgueira en el V alle del Rosal. Formalización de terrazas. Elaboración propia ${ }^{11}$.

Se estudia la aldea de A Burgueira, entendiéndola como elemento paradigmático y generalizable, de las zonas altas de los valles, situación que se da en múltiples pequeños valles en toda Galicia y de las tierras del noroeste peninsular adyacente. Se sitúa en un pequeño valle, el Valle del Rosal, a los pies de una sierra litoral, A Serra da Groba. Este hecho puede servir de referencia como formalización de territorios "aterrazados" para gestionar la

\footnotetext{
9 A. Martínez Cortizas, A. Pérez Alberti, Atlas Climático..., 128.

10 A. VAN DEN DRIES, The art of irrigation. The development, stagnation and redesign of farmermanaged irrigation systems in northern Portugal, Wageningen 2002, 37.

11 Contrasta el espacio cultivado de las terrazas con las zonas altas donde el espesor de la tierra vegetal es muy escaso y el granito aflora por todas partes.
} 
erosión y conseguir la fertilidad en una zona con una pluviosidad muy alta, pero donde al mismo tiempo -debido a su situación al sur de Galicia y a las condiciones climatológicas- es necesaria la irrigación de estas terrazas de cultivo, dado el balance hídrico negativo en los meses estivales. Como indica Abel Bouhier:

"debido a la elevación relativamente marcada de las temperaturas estivales, se manifiesta una cierta propensión a la aridez que tiende a acentuarse desde el norte cara al sur [...] La parte sudoeste, sobre suelos filtrantes que derivan de arenas graníticas, el cultivo del maíz sólo es posible con la ayuda de un pequeño riego de verano [Lo que da lugar a una organización singular del espacio agrario] La idea a la que se llega es que, motivada por las necesidades urgentes ligadas a la relativa sequedad estival y hecha posible por la intervención de algunos factores favorables, de orden estructural y morfológico, la organización del regadío procedió sobre todo de una voluntad humana de acondicionamiento en sí misma inseparable de un grado muy alto de ocupación de las parcelas cultivadas, y que exigió la utilización de ciertas técnicas menos fáciles de manejar de lo que a primera vista se podría pensar, y una organización peculiar del espacio agrario"12.

La formalización de estos territorios "aterrazados" debe responder por un lado a la capacidad de drenaje y a la capacidad de irrigación, acompañadas de una compleja gestión desde la aldea para adaptarse a la variabilidad de la naturaleza. El territorio escogido se ha de entender como un espacio que tendría todas las condiciones naturales para ser difícilmente habitable, sin embargo se trata de un espacio antropizado, ocupado desde antiguo, donde se ha creado un hábitat partiendo de la sabia gestión de un recurso como el agua. La recopilación y comprensión del sistema de conocimiento local para el manejo del medio en el caso concreto del agua, nos permite comprender las reglas de construcción del paisaje, herramientas básicas tanto para la

12 A. Bounier, Galicia: Ensaio xeográfico de análise e interpretación dun vello complexo agrario, Santiago de Compostela 2001, 63, 70 y 630.

"Los caminos del agua y la construcción de terrazas agrícolas..." 
conservación del paisaje rural como para el planteamiento de su gestión, que haga factible su perduración en el tiempo.

\section{Mapeando los múltiples caminos del agua}

Se ha puesto el foco sobre los tramos altos de los pequeños y abundantes valles, donde las condiciones iniciales para emplazar asentamientos poblacionales son más difíciles y donde la construcción de terrazas para el cultivo es quizá la única forma de sostener una población estable, una aldea. Para su estudio, se parte de la elaboración de una cartografía que permita representar no solo las construcciones, los caminos, las parcelas y las pendientes, sino entender y representar con fidelidad la geografía del agua, cartografiar el agua como vehículo para propiciar la vida. Se trabaja con un modelo digital del terreno -con una malla 05 -del Instituo Geográfico Nacional $(\mathrm{IGN})^{13}$ mediante software GIS (Geographic Information Systems) que permite reflejar todos los pliegues y micropliegues de un territorio extremadamente ondulado, propiciando una lectura a escala de detalle de las pequeñas formas que una cartografía convencional no admite. En esta representación geográfica meramente física, se superponen varios inputs, ya que el objetivo buscado es la representación del paisaje como un producto cultural, generado por la actividad humana.

Por un lado, se utilizan los datos ofrecidos por el trabajo "Proxecto Toponimia de Galicia" de la administración regional, donde, siguiendo el método antropológico, la microtoponimia de los territorios es recogida por medio de encuestas orales. La media de topónimos por $\mathrm{km}^{2}$ que se obtuvo fue de 50 , llegando a un máximo de 160 topónimos por $\mathrm{km}^{2}$, lo que da una idea de la tremenda intensidad de las singularidades territoriales nombradas

13 http://centrodedescargas.cnig.es/CentroDescargas/catalogo.do?Serie=LIDA2

14 Proxecto Toponimia de Galicia (PTG). Iniciado en el año 2000, está promovido por la Comisión de Toponimia de la Consellería de Presidencia, Administracións Públicas e Xustiza. 
por el hombre en el país gallego ${ }^{15}$. Su verificación e interpretación, permitió descubrir elementos ocultos, así como usos tradicionales olvidados; en palabras de Tilley, "fundamentalmente, los nombres crean paisajes"16. En cualquier espacio local para la comunidad que lo habita todo tiene un nombre, y así, en la parte superior del valle, para un área de estudio de casi $50 \mathrm{~km}^{2}$, se recogieron 5.903 topónimos, 122 topónimos por $\mathrm{km}^{2}$, de los cuales 909 son hidrónimos, 15\% del total con una relación de 19 hidrónimos por $\mathrm{km}^{2}$.

A partir de la microtoponimia y su verificación con el trabajo de campo, fue posible clasificar estas corrientes de agua en continuas -o permanentes -y discontinuas, que solo aparecen en momentos de lluvia. Las continuas son denominados regueiros, y regueiras, las discontinuas u ocasionales. Corrientes naturales, continuas y discontinuas, facilitan el drenaje del territorio, a las que se suman las corrientes manejadas, los regos y levadas que permiten la distribución y reparto del agua, con una geometría que se complementa y ramifica en sentido contrario en un esfuerzo por no perder cota y tratando de alcanzar las tierras y plantaciones más alejadas. Este trazado permite hacer visible el uso del agua y poner de manifiesto la relación entre las terrazas, las aldeas y la gestión del territorio desde el punto de vista del agua.

Para la representación del agua manejada, se ha recurrido a los datos proporcionados por una de las fuentes catastrales históricas que tiene una representación gráfica fiable -fotografía aérea muy precisa del llamado Vuelo Americano de 1956 -y que coincide con un momento álgido de desarrollo, el más reciente de la vida campesina pre-industrial en la zona rural de Galicia. Sobre las fotografías del "vuelo americano" la administración del

\footnotetext{
15 V. Feijoo ARES, "Nuevas aplicaciones para recoger, normalizar y difundir la toponimia de Galicia. Estado de los proyectos", Boletín de la Real Sociedad Geográfica, CLII, 2017, 91-126, 92.

16 C. Tilley, A phenomenology of landscape. Places, paths and monuments, Oxford 1994.
}

"Los caminos del agua y la construcción de terrazas agrícolas..." 
momento ha trabajado el parcelario y los usos del suelo. Este es el Catastro de la Riqueza Rústica del año 1956, donde a la representación gráfica de las parcelas se le añaden los datos de usos, superficies y propietarios, que son tratados y manejados como información alfanumérica para incorporar al GIS. Además del detalle y la información valiosísima que facilita, corresponde a un momento en pleno uso, a diferencia de la situación actual donde un buen número de parcelas han dejado de cultivarse $\mathrm{y}$, por tanto, de regarse. La alarmante despoblación que está sufriendo el mundo rural gallego ha dejado, a día de hoy, 3.860 aldeas abandonadas. La tendencia en los años venideros es superar esa cifra, pues tenemos 2.122 aldeas con una sola persona censada y 2.500 con dos habitantes, normalmente, personas mayores $^{17}$.

El Catastro de la Riqueza Rústica del año 1956 aporta para cada una de las parcelas: toponimia que incluye el nombre o paraje donde se sitúa la parcela, nombre del propietario o propietarios, tabla con la indicación de cultivo, aprovechamiento, edificios, pozos, fecha de las plantaciones, etc., clase local y extensión, incluyendo también la extensión del espacio comunal. Para conocer los datos de las parcelas regadas y no regadas se ha partido también de los datos obtenidos en esta fuente. Los datos de parcelas irrigadas y no irrigadas que, aunque sí están disponibles en otras partes de España a través de la aplicación informática SIGPAC, en el caso de Galicia, donde la aplicación es el SIXPAC ${ }^{18}$, no son públicos. Debido a dos causas, en Galicia dichos datos no son determinantes a la hora de pedir ayudas y además a causa de la gran cantidad de parcelas y lo reducido de sus dimensiones sería necesario otra aplicación informática para poder cargar tal densidad de información.

\footnotetext{
17 Fuente: Instituto Galego de Estatística https://www.ige.eu/

18 SIXPAC. El Sistema de Información Xeográfica de Parcelas Agrícolas es una base de datos oficial de referencia para la identificación de parcelas agrícolas. Su utilización es obligatoria en España para realizar las solicitudes de ayudas directa de la PAC que requieren declarar superficies https://sixpac.xunta.es/
} 
Los datos facilitados por el Catastro de la Riqueza Rústica, que reflejan un momento "maduro" de funcionamiento de una "agricultura tradicional", fueron verificados, complementados y ampliados con un trabajo de campo meticuloso realizado desde el año 2011 hasta el año 2015. Esta verificación consistió en una "observación participante" durante todo el período tanto de "riego de invierno" como de "riego de verano": es decir, y en palabras de Fabienne Wateau, en acompañar a los vecinos en las tareas de regar, participar en los trabajos comunitarios de mantenimiento de la infraestructura, recopilar información sobre el funcionamiento de las presas, recopilar los "libros del agua", preguntar por los repartos, etc. El riego se practica durante todo el año, tanto en verano como en invierno. La variabilidad en la disponibilidad de agua es muy grande a lo largo del calendario agrícola, muy abundante en invierno y otoño; en verano, sin embargo, se produce un período de estiaje. El riego de invierno se extiende en el campo de estudio desde el 8 de setiembre hasta la noche de San Juan. En otras partes del Minho portugués, las fechas de inicio y fin del período de riego de invierno también coinciden con las del área de estudio $^{19}$. En el invierno, el método y procedimiento de riego son adecuados para el transporte y aplicación de grandes cantidades de agua, es un trabajo extensivo. El uso del agua en invierno está íntimamente ligado a una función de fertilización y también a una función de protección. Como indica José Portela:

"en los regadíos tradicionales el agua alimenta a las plantas en el verano y las calienta en el invierno, permitiendo la obtención de producciones forrajeras" 20 .

El riego de invierno tiene también una función de favorecer la recarga larga de acuíferos durante la época de agua abundante para que ésta esté disponible en forma de numerosos

${ }^{19}$ F. Wateau, Conflictos e Água de Rega. Ensaio sobre a Organiz̧açao Social no Vale de Melgaço, Lisboa 2000, 27 y 44.

20 J. Gandra PORTEla, Os regadios tradicionais em Tras-os-Montes: a agua e o homem, Santiago de Compostela 1991.

"Los caminos del agua y la construcción de terrazas agrícolas..." 
manantiales durante la primavera. El riego de verano se extiende desde el 24 de junio, día de San Juan, hasta el 8 de setiembre. Si el resto del año cualquier parcela puede ser irrigada, siempre que por posición topográfica sea posible, durante el verano cuando el agua escasea, el ámbito de irrigación está limitado a las parcelas que tienen los derechos consuetudinarios para ello reflejados en los "libros de agua". Comienza el xiru y las rodas, principios de rotatividad para la distribución del agua, basados en la horizontalidad, la solidaridad y la equidad:

"La "roda" constituye un verdadero principio estructural de organización y reparto, con efectos de estructuración espacial, social, ideológica"21.

Finalmente, otra valiosa información fue incorporada. La única información escrita que refleja las distribuciones temporales para disponer de agua en las parcelas como el derecho al riego recogido en los "libros de agua", complementada por la investigación oral entre los habitantes de más edad -que siguen conservando las "reglas de manejo" y son depositarios de la memoria colectiva -que permitió recopilar e incorporar información no escrita sobre los estándares de gestión del agua, transmitida de generación en generación y readaptadas mil veces, manteniendo su esencia de distribución proporcional a la superficie de las parcelas con derecho a riego, de un recurso escaso, el agua. Una información esencial para comprender las reglas de uso y convivencia, fomentada y mantenida en la memoria local. Así, la representación de las terrazas de cultivo y del movimiento del agua responden tanto a un exhaustivo trabajo de campo territorial como a la comprobación y verificación de las "reglas del manejo" con los labregos de la aldea. Como hace saber Miguel Barceló:

${ }^{21}$ F. Wateau F, Conflictos e Água ..., 90. 
"el espacio irrigado no tiene sólo una identidad tecnológica sino que es sobre todo una opción social [...] La explicación del diseño del espacio hidráulico que sólo, es, finalmente, comprensible a partir del conocimiento de la organización social que lo ha producido"22.

La cartografía para la elaboración de los planos (Figuras 6, 7, 8, 9 y 10), sobre las capas base de información obtenidas de fuentes oficiales, se enriqueció con información y datos recogidos "in situ" tanto de trabajo de campo, como de fuentes orales así como de la única información escrita existente "los libros de agua": esto es, anotaciones donde se reflejan los repartos fundamentales para comprender el recorrido del agua en el xiru y en las rodas.

\section{Las "terrazas" del territorio cultivado}

En las tierras más bajas en relación a la aldea -emplazada a media ladera -se disponen las tierras de cultivo. Se formalizan en pequeñas superficies "aterrazadas" que escalonan las pendientes, a veces muy acusadas, para facilitar su laboreo y la infiltración del agua evitando la erosión del sustrato de tierra fértil. Todo este sistema de terrazas se ha ido creando a lo largo de la historia comenzando en las proximidades de los castros, práctica difundida paulatinamente durante el periodo romano-suevo, y adoptada con intensidad desde el medievo.

Así pues, en las tierras bajas de las aldeas se construyen bancales y terrazas, por simple acumulación de tierras con un mínimo soporte de vegetación arbustiva enraizada, o construyendo muros de contención, socalcos, con piedras eliminadas por molestas -de las tierras a cultivar. Se escalonan intencionadamente de esta forma porciones de terreno que facilitan el laboreo, la infiltración del agua de lluvia y evitan la erosión del sustrato fértil, identificando y reconociendo

22 M. Barceló, H. Kirchner, C. Navarro, El agua que no duerme. Fundamentos de la arqueología bidráulica andalusí, Granada 1996, 51.

"Los caminos del agua y la construcción de terrazas agrícolas..." 
subcuencas y microcuencas en el terreno que de este modo se antropizan para construir un paisaje cultural relevante.

La construcción y disposición de estas "terrazas" responde al entendimiento y comprensión culturales y comunitarios del agua en el territorio, de sus caminos naturales que se identifican y adecuan, de su papel esencial en la vida y en la fertilidad de los campos, de las distintas formas en que se puede obtener de la naturaleza en forma de lluvia directa, drenaje desde las tierras altas, y captación desde los cauces hídricos o manantiales buscados. Y así, se emplazan identificando pequeños pliegues topográficos, y se construyen perpendiculares a las escorrentías, de manera que los arrastres de arenas, limos, arcillas y materia orgánica en descomposición se detienen para formar suelo y fertilizar las tierras de cultivo en un sistema orgánico ${ }^{23}$ que se complementa con los paulatinos y anuales aportes de estiércol de las camas de los animales estabulados.

Al mismo tiempo, la necesidad de facilitar el movimiento del agua por gravedad para la irrigación hace necesaria la construcción de complejos sistemas ensamblados que permiten el paso del agua de unas a otras dotándolas de una ligera y medida pendiente que facilite tanto la entrada del agua de riego, como el drenaje en el momento de las lluvias. Las terrazas irrigadas se aproximan a la horizontalidad, pero siempre con una ligera pendiente $-3-5 \%$ en la dirección transversal, $0,7-2 \%$ en la dirección longitudinal -que permite que el agua de lluvia no se detenga y garantiza que el agua de riego pueda movilizarse para el efectivo riego de los cultivos. Además, los socalcos realizados con piedras en seco son drenantes, y de esta forma, el nivel freático nunca alcanzará la superficie cultivable de la tierra.

23 A. Chuchí, E. Albareda, R. Teira, E. Castro, D. Alba, N. Rigau, Estudio de las bases y el alcance de una Estrategia Verde para Santiago de Compostela, Barcelona 2010. 

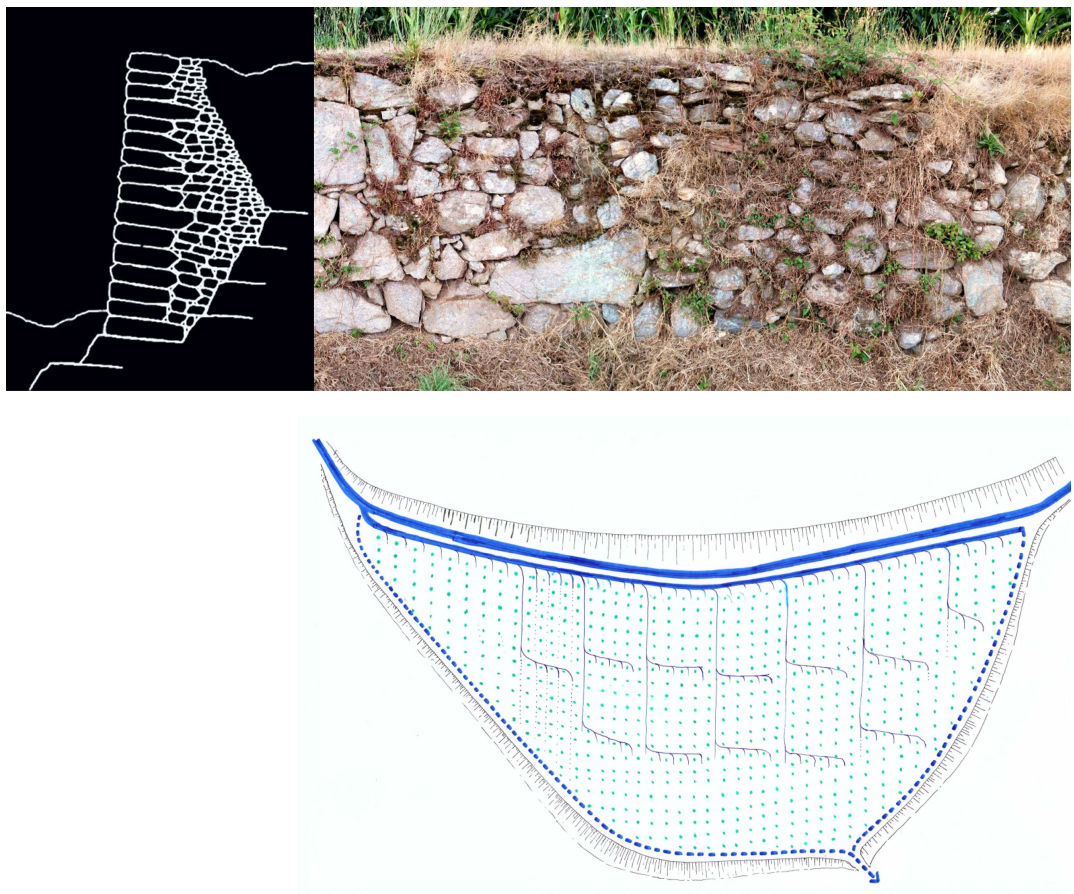

Fig. 3. Ejemplos de aliados de muros de contención, canales de riego y de drenaje. Elaboración propia ${ }^{24}$.

La contención se realiza con muros de piedra en seco. La altura de los muros de contención es variable en la zona de estudio. Las alturas más comunes son entre 1 y 3 metros. La altura depende de la inclinación de la pendiente y separación de los muros de contención. Cuando la pendiente es más pronunciada esta separación disminuye, siendo la separación más

\footnotetext{
${ }^{24}$ En la parte superior de la imagen alzado de muro de contención que constituyen las terrazas, realizadas con muros de piedra en seco de diverso tamaño. A la izquierda sección transversal del muro donde se observa hacia el interior la utilización de piedras pequeñas a modo de drenaje. En la parte inferior se representan tanto los canales de riego como los de drenaje en una terraza. Las terrazas irrigadas se aproximan a la horizontalidad, pero siempre con una ligera pendiente (3-5\% en la dirección transversal, $0,7-2 \%$ en la dirección longitudinal) que permite que el agua de lluvia no se detenga y garantiza que el agua de riego pueda movilizarse para el efectivo riego de los cultivos.
}

"Los caminos del agua y la construcción de terrazas agrícolas..." 
habitual entre terrazas entre 12 y $30 \mathrm{~m}$. En los muros de contención que constituyen las terrazas, hacia el interior de la misma es habitual utilizar piedras pequeñas a modo de drenaje, colocadas a medida que se va ejecutando el muro, que se interpone entre el interior de la pared y el suelo de relleno. El muro suele tener una pequeña inclinación en relación a la vertical, que contrarresta el empuje del terreno y que depende de la altura de la pared y del tamaño y calidad de las piedras que lo componen. En cuanto al aparejo, se trata de piedras muy irregulares de diversos tamaños, aunque predominan las de pequeño tamaño (Vid. Fig. 3).

\section{La construcción responde a "la geometría del agua" 4.1 Las terrazas}

Para adaptarse a la topografía los muros que soportan las terrazas se construyen perpendicularmente a las regueiras, -corrientes estacionales debidas al régimen de lluvias que la cartografía GIS permite visibilizar-, deteniendo la escorrentía. Es fácil suponer que para la acumulación de este terreno fértil, la escorrentía de las aguas de lluvia juega un papel esencial. El agua propicia el arrastre de suelos -limos, arcillas y arenas -y materia orgánica y la intencionada construcción de muros de piedra facilita la deposición, acumulación y fijación de estos suelos fértiles, consiguiendo la reducción de los volúmenes de flujo y obteniendo además superficies más horizontales para el cultivo y un control de la fuerza erosiva del agua.

Se observa, en toda esta construcción, un conocimiento detallado de las características de la escorrentía que da lugar a la aparición de presas, pequeños depósitos de agua para el riego, intercaladas entre algunas de las terrazas. Esta agua, que es beneficiosa, puede ser también perjudicial para los cultivos, de ahí que la previsión de drenajes en estas piezas sea también muy importante $\mathrm{y}$, en la construcción de los abancalamientos, en muchas ocasiones se disponen canos, drenajes hechos con piedras, 
por donde se favorece que el agua discurra. Por lo tanto, la lectura del agua es clave para entender su formalización.
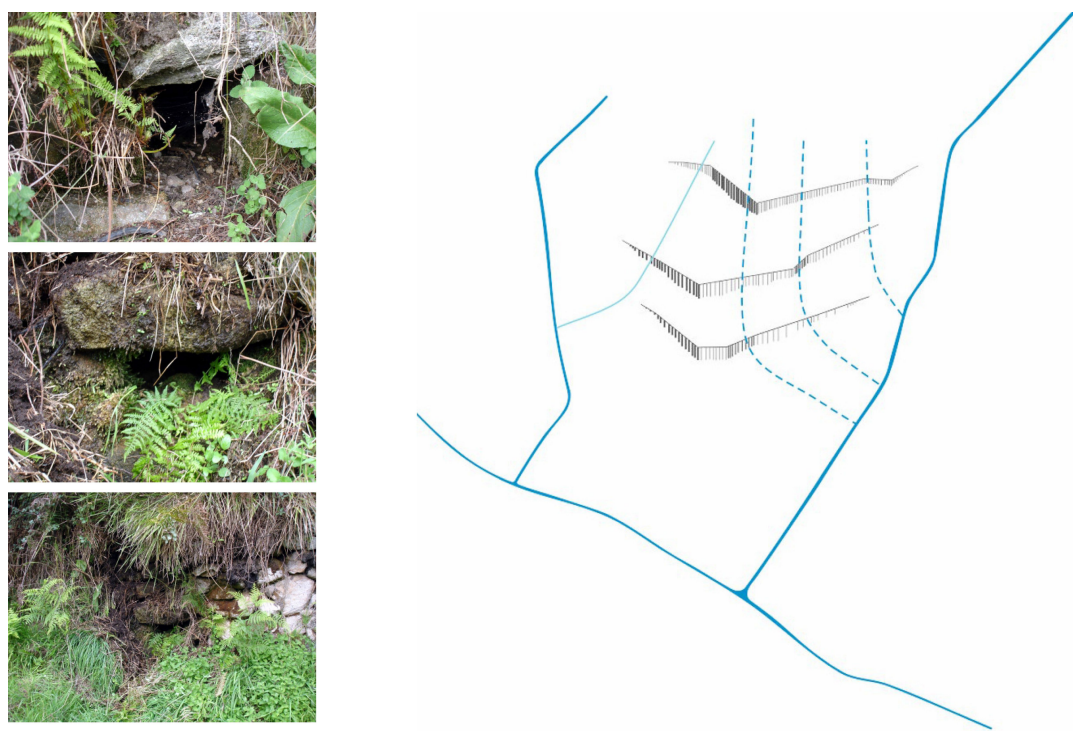

Fig. 4. Sistemas de construcción de las terrazas. Elaboración propia ${ }^{25}$.

\subsection{Los caminos del agua}

Hay dos características hidrológicas de los recursos hídricos que son muy importantes para la comprensión del sistema: por un lado, la alta variabilidad del agua a lo largo del año y la alta interconectividad de los recursos hídricos. Precisamente en esta interconectividad se apoya la construcción de la red de irrigación. Como se comprueba más adelante, las distintas presas y levada

\footnotetext{
25 Las terrazas se construyen respondiendo al agua, se disponen perpendiculares a las regueiras (corrientes estacionales debidas al régimen de lluvias), deteniendo la escorrentía; con respecto a las corrientes pequeñas continuas se disponen canos (drenajes hechos con piedras que se disponen en las terrazas para facilitar el drenaje, en la parte izquierda fotografías de varios canos); los regueiros (corrientes de agua continuas) separan lotes y sirven como puntos de drenaje de los regos fureiros (red de drenaje que conduce las aguas broncas a la red cóncava natural). Se comprueba como es el agua el principio ordenador del diseño.
}

"Los caminos del agua y la construcción de terrazas agrícolas..." 
están acopladas entre ellas permitiendo el reabastecimiento óptimo del sistema.

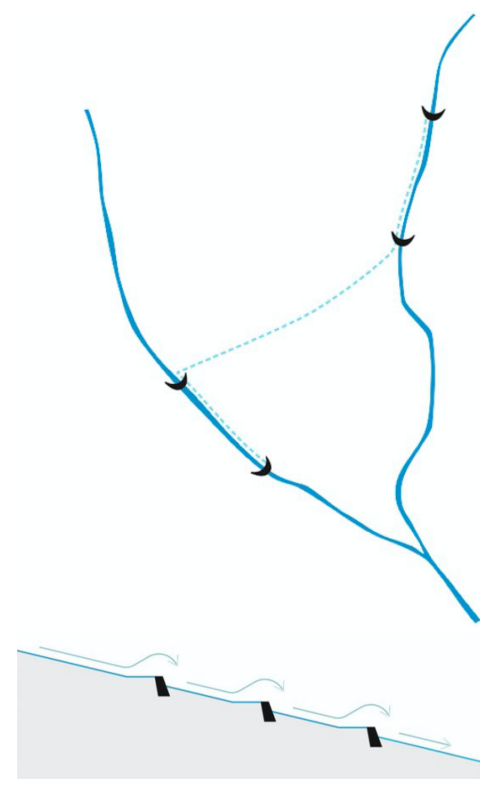

Fig. 5. Red de irrigación. Elaboración propia ${ }^{26}$.

Se trata de un espacio que para ser habitado debe hacer frente por un lado -debido a las fuertes pendientes y los montes que lo rodean -a resolver los problemas provocados por las fuertes escorrentías y, por otro, -por las condiciones climáticas y falta de retención de humedad del suelo- a habilitar la irrigación de muchas de las tierras de cultivo en los meses estivales.

\footnotetext{
26 Se trata de un conjunto coherente, en el que los distintos elementos, presas y levada, se acoplan entre sí. El rebose alimenta a las presas situadas aguas abajo, incluso permitiendo el trasvase entre dos corrientes continuas. El agua está en continuo movimiento y uso: "el agua no duerme".
} 
A. SAntos VÁzQuez, H. SEOANE, C. Martínez GonzÁLEZ

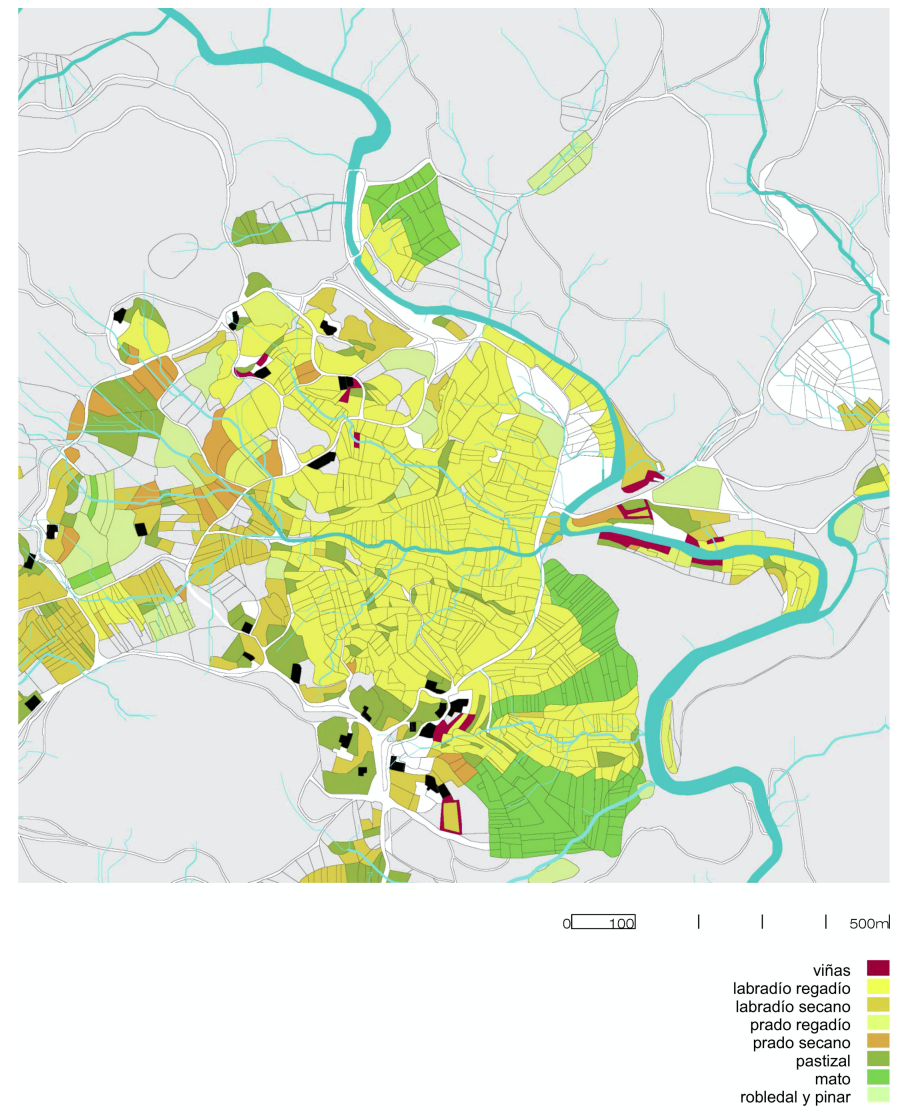

Fig. 6. Aldea de A Burgueira en el V alle del Rosal. Parcelario y cultivos con indicación de parcelas irrigadas. Elaboración propia ${ }^{27}$.

Se compone de una red cóncava natural de regueiros y regueiras que va drenando y añadiendo caudal aguas abajo, la red natural del agua que aflora y las aguas de lluvia.

27 A partir de la restitución del parcelario de la Cartografía Catastral actual con apoyo en el Catastro de Riqueza Rústica 1956.

"Los caminos del agua y la construcción de terrazas agrícolas..." 


\section{RIPARIA VOL. 6 (2020)}

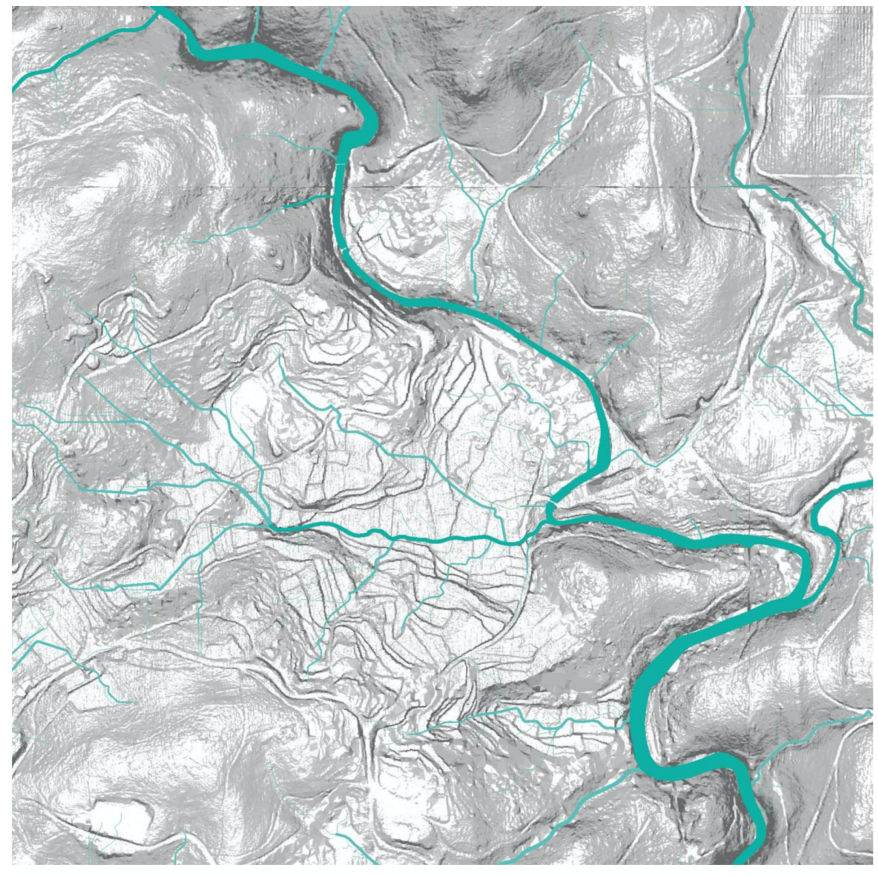

Fig. 7. Aldea de A Burgueira en el V alle del Rosal. Formalización de terrazas en relación a las corrientes de agua. Elaboración propia ${ }^{28}$.

A esta se superpone una red convexa, el agua construida, formada por la levada, las presas, los canales y sus ramificaciones que va infiltrando y distribuyendo capilarmente el agua, y trata de homogeneizarla sobre el territorio a irrigar.

28 A partir de nube de puntos LIDAR del Instituto Geográfico Nacional (IGN). 


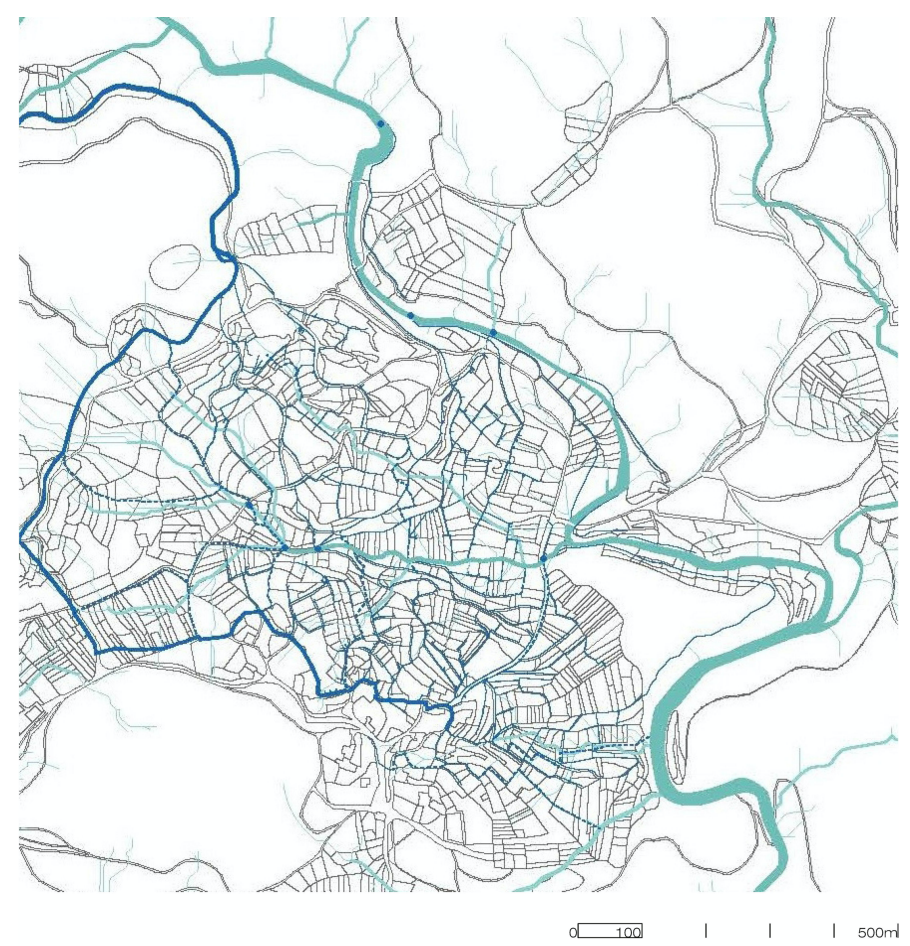

Fig. 8. Aldea de A Burgueira en el Valle del Rosal. Formalización de la red de agua. Elaboración propia ${ }^{29}$.

Estas dos redes se cruzan, se interconectan y a veces incluso se superponen, de difícil interpretación, pero, que con un sabio manejo, convierten un espacio inhóspito en habitable creando y construyendo paisaje. Además, si hablábamos de la red cóncava natural que se maneja desde el reconocimiento, existe

29 A partir de los "libros del agua" y trabajo de campo. Funcionará como red de riego y como red de drenaje cuando las aguas son abundantes adaptándose a la variabilidad de la naturaleza.

"Los caminos del agua y la construcción de terrazas agrícolas..." 
también otra red cóncava artificial que forman los denominados regos fureiros una red de drenaje imprescindible cuando el agua es un problema por su exceso. Red de escorrentía para que no invadan las aguas broncas conduciéndola a la red cóncava natural. Se antropizan y se domestican las aguas broncas.

\subsection{El sistema}

La representación detallada de todas estas venas de agua incluso las discontinuas pone en evidencia la relación entre las regueiras y las terrazas de cultivo. Las terrazas al escalonar espacios cultivables del territorio, con la finalidad de modificar la pendiente para reducir la erosión, facilitan la infiltración directa del agua de lluvia e incrementan la capacidad de retención de la humedad. Se observa como cuando las pendientes son altas no se "aterraza", solo en las zonas donde la escorrentía tiene una pendiente más baja. Cuando las pendientes de la escorrentía son superiores al $20 \%$ no se "aterraza", destinando estas tierras a monte o baldíos comunitarios.

Las corrientes continuas de agua, los regueiros que delimitan estas zonas cultivadas, se construyen hasta el límite con muros de piedra, buscando el máximo aprovechamiento de las zonas cultivadas. La búsqueda de la horizontalidad para el cultivo ha encajado estos regueiros para -buscando las estrecheces naturales -emplazar numerosas presas, pequeños depósitos de agua, desde donde regar esas terrazas, definiendo con su emplazamiento la cota de la línea máxima que puede alcanzar el agua de riego, a la que se irá superponiendo la siguiente y así sucesivamente, en una construcción puramente geométrica, donde las cotas de las terrazas vienen fijadas por el agua y la necesidad de su movimiento por la gravedad. De esta forma se genera un complejo sistema donde las terrazas se van engarzando entre ellas, para que el agua pueda pasar de unas a otras. 


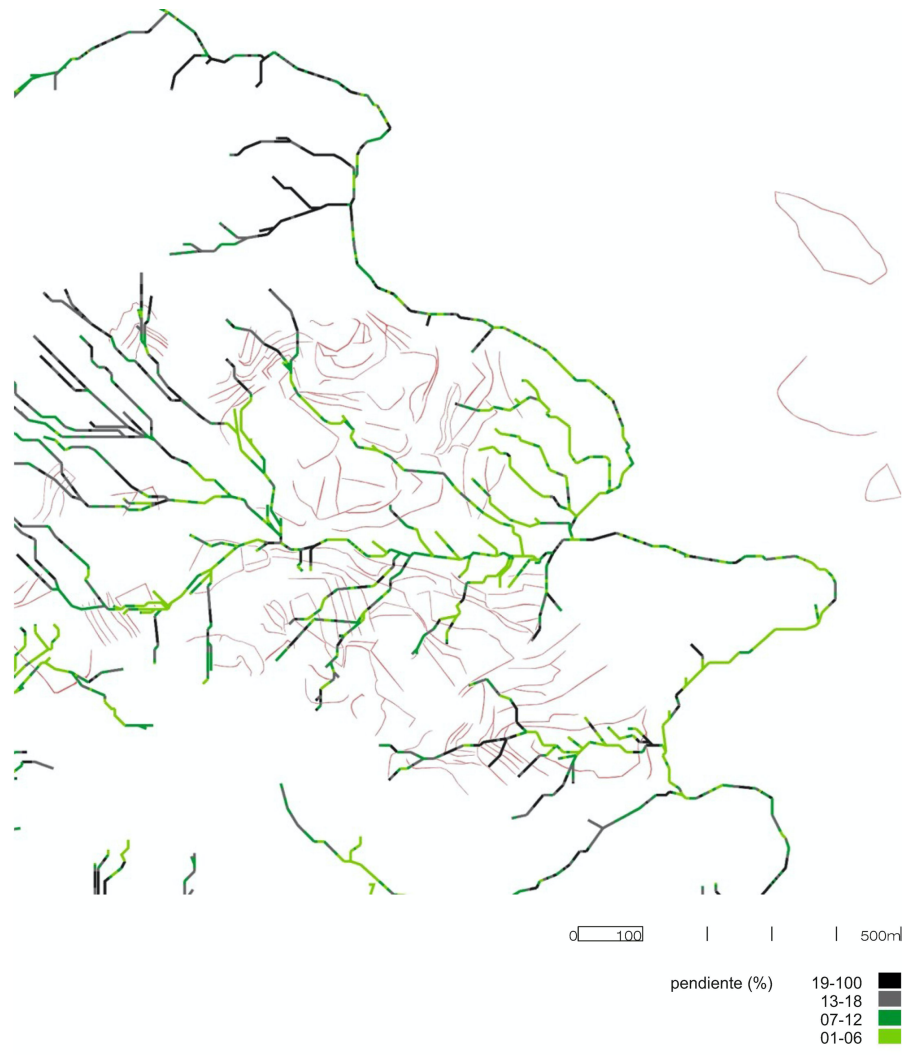

Fig. 9. Aldea de A Burgueira en el Valle del Rosal. Relación de los muros de las terrazas con la escorrentía del agua. Elaboración propia ${ }^{30}$.

Geométricamente se van definiendo unidades de riego factible. Se descubre un sistema absolutamente medido y

\footnotetext{
${ }^{30}$ A partir de la graduación de pendientes de la red hidrológica en base a información de modelo digital del terreno MDT05-LIDAR del Instituto Geográfico Nacional (IGN). Se observa que cuando las pendientes son altas - colores oscuros- no se aterraza, solo en las zonas donde la escorretía tiene una pendiente más baja.
}

"Los caminos del agua y la construcción de terrazas agrícolas..." 
construido según la estricta disciplina de las leyes de la hidráulica y con un dominio de la pendiente y un control de la velocidad del agua que asegure el movimiento de la misma, y que al mismo tiempo evite la erosión. Se pone así en evidencia la "naturaleza ultraestable" 31 de estos sistemas hidráulicos, como los denomina T. F. Glick, que permite distinguir, por debajo de materializaciones recientes, un diseño antiguo:

"Esta naturaleza estable de sistemas físicamente frágiles se basa en la invariabilidad de los puntos de captación del agua y el trazado y pendiente de los canales de derivación, la localización de las albercas, etc."32.

En general, muchos de estos elementos, como las presas en el río Tamuxe, eran elementos frágiles, fácilmente destruibles por las riadas en invierno, al que ofrecían poca resistencia, pero al mismo tiempo fácilmente reconstruibles en verano, cuando eran necesarias para regar. Hoy en día, han sido sustituidas por pequeños depósitos realizadas en hormigón en el río, pero situadas en el mismo espacio de las unidades originales, porque la rigidez del espacio hidráulico impone generalmente la estabilidad del emplazamiento de dichas unidades. En este caso, las técnicas y las formas constructivas son de gran sencillez. Con una gran economía de medios se elaboran un número limitado de soluciones, aunque con gran sofisticación en la adaptación a las condiciones del medio, aprovechando al máximo las condiciones naturales. Se trata en definitiva de un sistema muy sofisticado con pocos elementos, pero con una gran diversidad en la adaptación formal a las distintas particularidades de cada lugar.

Al comparar las cotas de las presas se verifica que están acopladas entre ellas y que la curva de nivel se hace visible a través de la formalización de las terrazas, de manera que los

31 T. F. GLICK, Regadío y sociedad en la V alencia medieval, Valencia 2003.

32 M. Barceló, H. Kirchner, C. Navarro, El agua que no duerme..., 59. 


\section{A. SANTOS VÁZQUEZ, H. SEOANE, C. MARTínEZ GONZÁLEZ}

"reboses" de las situadas aguas arriba alimentan a las situadas aguas abajo.
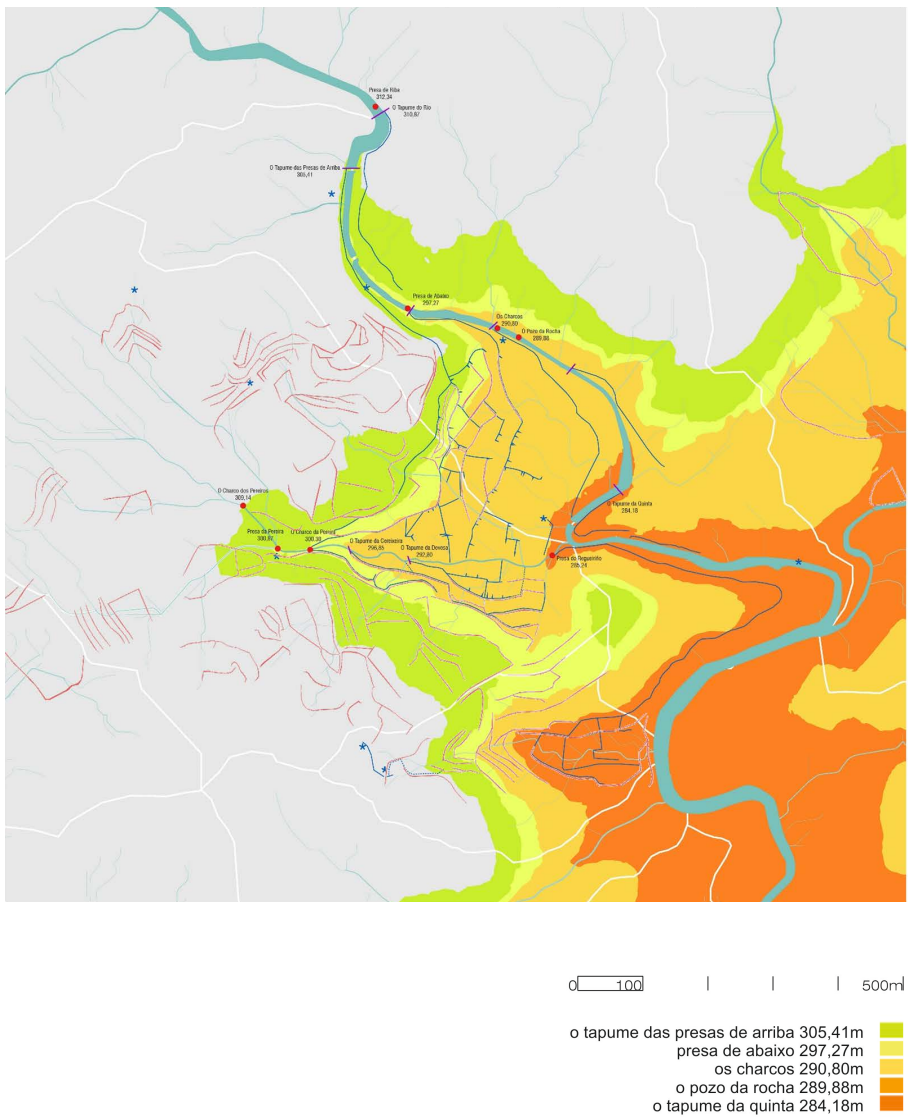

Fig. 10. Aldea de A Burgueira en el Valle del Rosal. Representación de cota. Elaboración propia 33 .

$33 \mathrm{Al}$ representar la cota, las presas revelan una localización relacionada con la altitud en secuencia descendente, secuencia que se hace visible en la sucesión de terrazas. A partir de altimetría en base a las altitudes de las presas obtenidas de modelo digital del terreno MDT05-LIDAR del Instituto Geográfico Nacional (IGN)

"Los caminos del agua y la construcción de terrazas agrícolas..." 
El "agua no duerme" en palabras de Miquel Barceló pues está en movimiento y uso constante. Se trata de sistemas como indica Patrice Cressier:

"que pueden sucederse a lo largo de un río o una rambla, con acoplamiento de uno a otro: el final de la acequia del sistema situado aguas arriba coincide con la captación del situado aguas abajo"35.

Se intuye que este acoplamiento y disposición de los elementos no puede ser casual -ni cuando se realizan al mismo tiempo, ni en previsión de implementaciones futuras -ya que además de permitir un reabastecimiento óptimo del acuífero, indican una organización social propicia para el establecimiento de pactos respecto a la gestión del agua y la construcción de la infraestructura.

\section{Conclusiones}

Cada aldea, con su territorio asociado, constituye un espacio hidráulico que se ha materializado moviendo tierras y manipulando terrenos y especialmente construyendo caminos para el agua para minimizar sus efectos perversos en la abundancia y gestionar su reparto en la escasez, adaptándose en su administración a su ciclo natural, y generando en definitiva un notable y singular paisaje cultural que responde al manejo del agua. Además, el agua utilizada por la comunidad aldeana es entendida culturalmente como un bien común ${ }^{36}$. Un bien necesario que se comprende en su ciclo. Un medio, un vehículo de fertilización que ofrece la naturaleza, un bien en movimiento constante, que se usa y se libera de nuevo en el ciclo a otros regantes. Toda la infraestructura está planteada para favorecer la recarga de acuíferos, y para servir a otros regantes o a otras aldeas

\footnotetext{
34 M. Barceló, H. Kirchner, C. Navarro, El agua que no duerme...

35 P. Cressier, La maîtrise de l'eau en al-Andalus Paysages pratiques et techniques, Madrid 2006.

36 E. Ostrom, El gobierno de los bienes comunes. La evolución de las instituciones de acción colectiva, México, 2011, 165.
} 
aguas abajo en el valle. En este sentido, el agua no se consume, simplemente se usa pues como ya se dijo, el agua no duerme ${ }^{37}$, y una vez realizada la función de portadora de nutrientes para los cultivos, infiltrándose en las tierras de las terrazas, proseguirá su camino aguas abajo, captándose de nuevo por otras comunidades aldeanas de regantes manteniendo en uso continuo el recurso.

El paisaje agrícola se construye, se conforma en terrazas, en una serie de planos sensiblemente horizontales que facilitan el laboreo agrícola. Se construyen teniendo en cuenta el agua presente o latente, y los muros que las conforman se disponen perpendiculares a las escorrentías o regueiras naturales, precisamente para propiciar la formación y retención de suelo fértil y más profundo, y así facilitar la infiltración de agua de lluvia, incrementar la retención de la humedad y evitar la erosión superficial. Los conjuntos de terrazas se separan por los regueiros, corrientes continuas, que junto con los regos fureiros forman la red de drenaje que se maneja desde la comunidad como forma de evitar que la acumulación del agua de lluvia pueda ser dañina para los cultivos. Todo hace suponer que el proceso histórico de sedentarización se hizo posible acompañado de la paulatina construcción de terrazas, desde una percepción integral del hinterland de cada asentamiento y desde la observación de que allí donde se producía la formación espontánea de tierra fértil, debido a los arrastres que acompañan a las escorrentías, podían generarse tierras de cultivo.

A medida que se dominan los procesos de fertilización y se mejora la calidad de las tierras con la incorporación del estiércol, la construcción de estructuras para el manejo del agua permite extender sucesivamente las áreas cultivables y conseguir cosechas también en tiempo de verano, completando un complejo sistema cuya finalidad es el cultivo intenso de las tierras de la aldea.

37 M. BARCEló, H. KirChner, C. NAvarro, El agua que no duerme...

"Los caminos del agua y la construcción de terrazas agrícolas..." 
En definitiva, toda la construcción del territorio se pone al servicio del mantenimiento del agua como vehículo de fertilización de las tierras y tanto la morfología de terrazas y baldíos como las redes de canales, regos fureiros, regueiras y reguieros, presas y desde luego la levada funcionan conjunta, cohesiva y solidariamente para la consecución del fin propuesto, todo sabiamente administrado por los habitantes de la aldea de una forma comunitaria. 


\section{Bibliografía}

M. BARCEló, H. Kirchner, C. NAvArro, El agua que no duerme. Fundamentos de la arqueología Hidráulica andalusí. Maracena, Granada 1996.

A. BOUHIER, Galicia: Ensaio xeográfico de análise e interpretación dun vello complexo agrario., Santiago de Compostela 2001.

J. Ma CARDESÍN, Tierra, trabajo y reproducción social en una aldea gallega (S. XVIII-XX), Bilbao 1992.

F. Carreras y CANDI F. (1928-1935), Geografia General del Reino de Galicia, 6 vols., La Coruña 1980.

P. CRessier, La maîtrise de l'eau en al-Andalus. Paysages, pratiques et techniques, Madrid 2006.

A. Cuchí Burgos, E. Albareda, R. Teira, E. Castro. D. Alba, N. RIGAU, Estudio de las bases y el alcance de una Estrategia Verde para Santiago de Compostela, Barcelona 2010

V. FEIJOO ARES, "Nuevas aplicaciones para recoger, normalizar y difundir la toponimia de Galicia. Estado de los proyectos", Boletín de la Real Sociedad Geográfica, CLII, 2017, 91-126.

J. GANDRA PORTELA, Os regadios tradicionais em Tras-os-Montes: a agua e o homem, Santiago de Compostela 1991.

T. F. GLICK, Regadio y sociedad en la Valencia medieval, Valencia 2003.

E. HERMON, "L'evolution diachronique des concepts environnementaus des deux rives de l'Atlantique. La construction du concept de RIPARIA comme un patrimoine culturel", RIPARIA 1, 2015, 1-20.

P. LAUREANO, Atlas de agua. Los conocimientos tradicionales para combatir la desertificación, Barcelona 2005.

A. Martínez Cortizas, A. PÉrez Alberti, Atlas Climático de Galicia. Santiago de Compostela, 1999.

J. R. MENÉNDEZ DE LUARCA, La construcción del territorio. Mapa histórico del noroeste de la península ibérica, Madrid 2000.

E. OSTROM, El gobierno de los bienes comunes. La evolución de las instituciones de acción colectiva, México 2011.

"Los caminos del agua y la construcción de terrazas agrícolas..." 
RIPARIA VOL. 6 (2020)

J. R. Raposo, J. Molinero, J. DAfonte, "Parameterization and quantification of recharge in crystalline fractures bedrocks in GaliciaCosta (NW Spain)", Hydrol, Earth Syst. Sci. 16, 2012, 1667-1683.

E. SEOANE, A ría como soporte da construcción da periferia urbana, A Coruña 2013. Tesis doctoral

C. TILLEy, A phenomenology of landscape. Places, paths and monuments, Oxford 1994.

VV. AA., Proxecto Toponimia de Galicia (PTG), https://toponimia.xunta.gal/gl/proxecto/antigo-ptg

A. VAN DEN DRIES, The art of irrigation. The development, stagnation and redesign of farmer-managed irrigation systems in northern Portugal, Wageningen 2002.

F. WhteAu, Conflictos e Água de Rega. Ensaio sobre a Organizaçao Social no $\checkmark$ ale de Melgaço, Lisboa 2000. 